\title{
Neural, Mechanical, and Geometric Factors Subserving Arm Posture in Humans ${ }^{1}$
}

\author{
F. A. MUSSA-IVALDI, N. HOGAN, ${ }^{*}$ AND E. BIZZI ${ }^{2}$ \\ Dcpartment of Psychology and * Department of Mechanical Engineering, Massachusetts Institute of Technology, \\ Cambridge, Massachusetts 02139
}

\begin{abstract}
When the hand is displaced from an equilibrium posture by an external disturbance, a force is generated to restore the original position. We developed a new experimental method to measure and represent the field of elastic forces associated with posture of the hand in the horizontal plane. While subjects maintained a given posture, small displacements of the hand along different directions were delivered by torque motors. The hand was held in the displaced positions and, at that time, we measured the corresponding restoring forces before the onset of any voluntary reaction. The stiffness in the vicinity of the hand equilibrium position was estimated by analyzing the force and displacement vectors.

We chose to represent the stiffness both numerically, as a matrix, and graphically, as an ellipse characterized by three parameters: magnitude (the area), shape (the ratio of axis) and orientation (direction of the major axis). The latter representation captures the main geometrical features of the elastic force field associated with posture. We also evaluated the conservative and nonconservative components of this elastic force field. We found that the former were much larger than the latter and concluded that the behavior of the neuromuscular system of the multiarticular arm is predominantly spring-like.

Our data indicated that the shape and orientation of the stiffness were invariant over subjects and over time. We also investigated the ability of our subjects to produce voluntary and adaptive changes in the stiffness. Our findings indicated that, when a disturbance acting along a fixed and predictable direction was imposed, the magnitude of the stiffness was increased but only minor changes in shape and orientation occurred. Taken together, all of these experiments represent a step toward the understanding of the interactions between geometrical and neural factors involved in maintaining hand posture and its interactions with the environment.
\end{abstract}

Received December 28, 1984; Revised April 5, 1985

Accepted May 2, 1985

\footnotetext{
${ }^{1}$ This research was supported by National Institute of Neurological Disease and Stroke Research Grant NS09343, National Institute of Arthritis, Metabolism, and Digestive Diseases Grant AM26710, and National Eye Institute Grant EY02621. F. A. M-I. is supported by a Consiglio Nazionale delle Richerche fellowship.

${ }^{2}$ To whom correspondence should be addressed, at Whitaker College, E25-526, Massachusetts Institute of Technology, Cambridge, MA 02139.
}

The studies reported in this paper are directed at understanding the processes subserving postural stability of a multi-joint limb such as the arm. Previous single-joint work on posture and movement showed that muscles, in vivo, have spring-like properties and that the stiffness at each joint is determined by the spring-like properties of activated musculature (Rack and Westbury, 1969, 1974; Nichols and Houk, 1976; Hoffer and Andreassen, 1981; Houk and Rymer, 1981). These observations suggested that postural stability results from the central nervous system (CNS) coordinating the activity levels of agonist and antagonist muscles around a joint so that an equilibrium position is defined (Feldman, 1966; Rack and Westbury, 1969, 1974; Bizzi et al., 1976, 1978, 1982a, b, 1984; Kelso, 1977; Cooke, 1979; Polit and Bizzi, 1979; Kelso and Holt, 1980).

Our goal in the investigations presented here was to develop these ideas in the context of multi-joint posture and, in particular, to characterize the net spring-like behavior of all the muscles in the arm. To deal with the richer and more complex situation of the multijoint arm, we have developed a new approach to the study of posture and movement. This approach entails displacing the hand in many different directions and each time determining the resulting restoring forces. We have developed a test of the extent to which the steady-state behavior of the neuromuscular system generating these forces is spring-like. This test, which is possible only in a multijoint system, allowed us to conclude that the neuromuscular system is predominantly spring-like. We also found that the displacement of the hand induced a restoring force with a static component that was related in both magnitude and direction to the displacement from the equilibrium position. We have developed a compact and mathematically concise representation of these directional properties in terms of the orientation, shape, and magnitude of the field of the elastic restoring forces. These parameters provide a new and richer description of posture. We have also studied the ability of the CNS to modulate and control these restoring forces by investigating the forces generated at the hand in a number of work space locations in normal human subjects. We found regularities in the shape and orientation of the field of spring-like forces, which indicate that the CNS actively coordinates the postural behavior of the arm by synergistic activation of all of the muscles.

\section{Materials and Methods}

Apparatus. Subjects were seated with the shoulder restrained by a shoulder harness belt, and the right, dominant hand gripped the handle of a two-link planar manipulandum (Fig. 1A). The wrist and the palm of the hand were bandaged with a calcium-impregnated gauze, which was also wrapped around the handle of the manipulandum to create a firm and rigid connection between the hand-wrist segment and the apparatus. The elbow was supported in the horizontal plane by a long rope $(3 \mathrm{~m})$ attached to the ceiling. A set of light-emitting diodes, used as visual targets, was mounted on a Plexiglas screen above the manipulandum. 

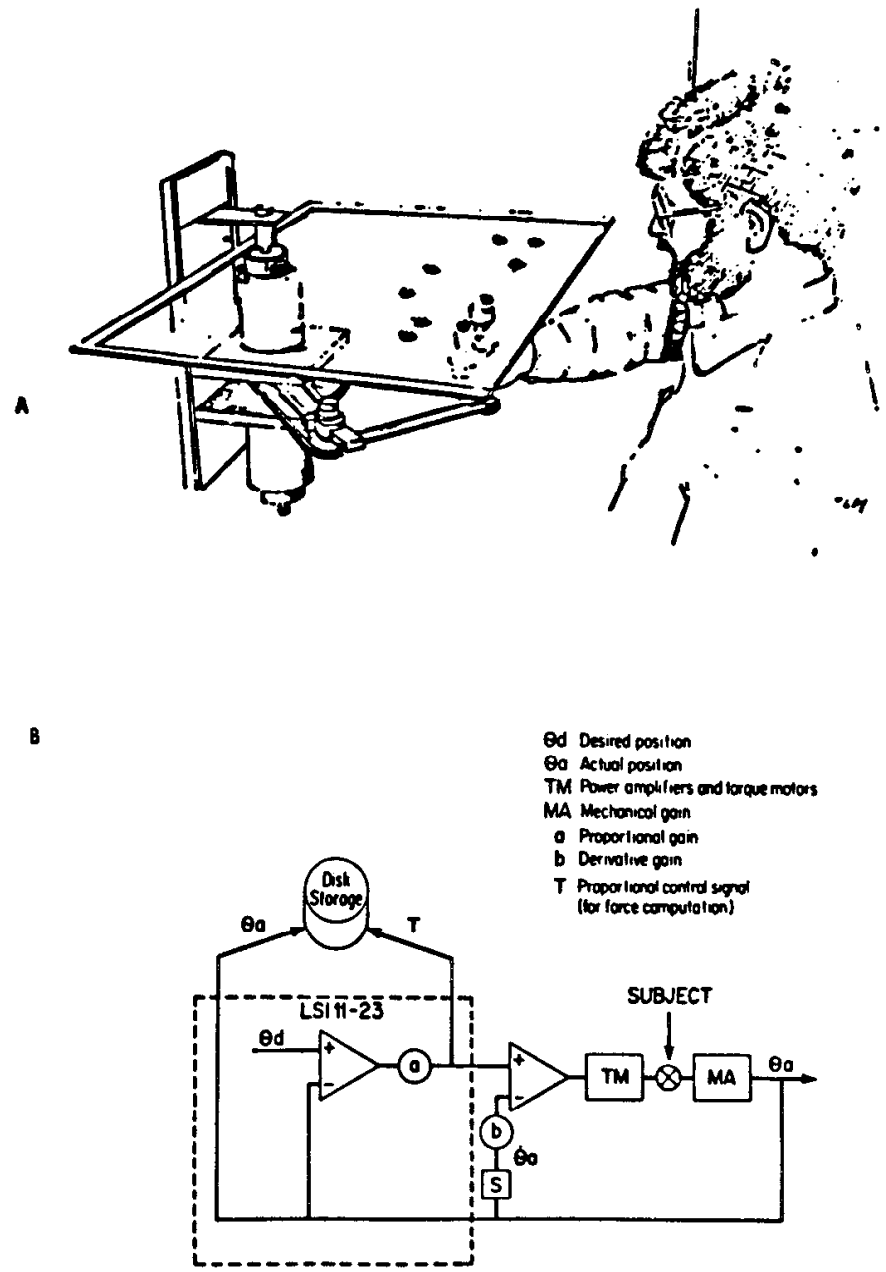

Figure 1. Experimental setup. $A$, Sketch of the apparatus in a typical experimental situation. $B$, Block diagram of the control scheme of the apparatus. The desired position vector, $\theta_{\alpha}$, is expressed in the joint anglo coordinates of the mechanical arm. $\theta_{d}$ is compared with the actual position $\theta_{a}$, and a proportional term, $a \cdot\left(\theta_{d}-\theta_{a}\right)$, is computed. This value is converted into an analogue signal from which a voltage, proportional to the aclual joint velocity, $\theta_{a}$, is subtracted. The resulting signal is fed to the torque motor. The subject acts as a mechanical disturbance that determines, together with the control signal, the actual position of the device. Both the actual position and the proportional signal are sampled and stored. The dashed line encloses the portion of the control process carried out digitally by an LSI 11/23 computer.

Two high resolution, 5-kilohm potentiometers (NEI-78ESC502) mounted on the axes of the mechanical joints were used to monitor the position of the hand in the horizontal plane. Two torque motors (E. G. G. Torque Systems: MH-3528 motors and C0501 transconductance servo amplifiers) were mounted at the base of the apparatus and were connected independently to each joint. This apparatus permitted us to apply to the handle a force of controlled amplitude and direction. A Digital Equipment Co. LSI-11/ 23 minicomputer was used to collect position data from the potentiometers and to control the torque motors in a servo mode, as indicated in Figure $1 B$. Each of the two mechanical joinls was controlled independently by using a linear position and velocity feedback law:

$$
T^{\prime}=a \cdot\left(\theta_{d}^{\prime}-\theta_{a}{ }^{\prime}\right)-b \cdot\left(\dot{\theta}_{a}{ }^{\prime}\right)
$$

where $T^{\prime}$ is the controlled torque, $\theta_{d}{ }^{\prime}$ is desired joint angle, $\theta_{a}{ }^{\prime}$ is the actual joint angle, and $\dot{\theta}_{a}^{\prime}$ is the actual angular velocity; $a$ and $b$ are constant feedback gains that can be set by the experimenter. They determine the effective "stiffness" and "viscosity" of the apparatus. The desired angles $\theta_{d}$ ' corresponding to a desired location of the end-point were computed by inverting the kinematic equations 3 for the manipulandum linkage.

The motion of the manipulandum was described in two cartesian coordinate frames: the subject's frame and the apparatus frame. The origin of the subject's frame was the center of rotation of the subject's shoulder, whereas the origin of the apparatus frame was at the intersection of the rotation axis of the motors with the horizontal plane. The $x$ and $y$ axes of the two systems were parallel to each other but were pointing in opposite directions. The subject's $x$ axis was lying in the frontal plane passing through the centers of rotation of both shoulders. The transformation from subject coordinates to apparatus coordinates is then given by

$$
\begin{aligned}
& x^{\prime}=x_{0 a}-x \\
& y^{\prime}=y_{o a}-y
\end{aligned}
$$

where $x_{0 \mathrm{a}}$ and $y_{\mathrm{oa}}$ are the coordinates of the apparatus origin in the subject's frame; $x$ and $y$ are the coordinates of a point in the subject's frame; and $x^{\prime}$ and $y^{\prime}$ are the coordinates of the same point in the apparatus frame. The inverse transformation from apparatus to subject coordinates is straightforward. The (desired) displacement was planned in the subject's coordinates and then translated to apparatus coordinates for control purposes. Conversely, the actual displacements were stored as apparatus coordinates and then converted into subject's coordinates.

Procedures and tasks. Ten subjects were tested. Each subject was asked to position the right hand under a particular visual target. In different runs, subjects placed the hand in five different locations. These locations will be referred to here as "reference" $(x=-5 \mathrm{~cm}, y=38 \mathrm{~cm})$, "distal" $(x=-5 \mathrm{~cm}$, $y=52 \mathrm{~cm}$ ), "proximal" $(x=-5 \mathrm{~cm}, y=24 \mathrm{~cm})$, "right" ( $x=28 \mathrm{~cm}, y=38$ $\mathrm{cm})$, and "left" ( $x=-40 \mathrm{~cm}, y=38 \mathrm{~cm})$. The postures of the upper arm and forearm corresponding to these five locations are schematically indicated in Figure 7. All of the coordinates of these points are expressed with respect to the subject's shoulder.

To avoid possible artifacts arising from changes in the configuration of the apparatus, we always kept the handle at the same position relative to the apparatus and changed the subject's position relative to the apparatus to test performance in different parts of the subject's work space.

When the hand was under a visual target, we applied a random sequence of displacements of variable magnitude and orientation. The displacement magnitudes were either 5 or $8 \mathrm{~mm}$, whereas the orientations were selected at random from a list of eight possible angles ranging from 0 to $325^{\circ}, 45^{\circ}$ apart. The movement following the onset of the disturbance from the manipulandum had a duration of about $120 \mathrm{msec}$ (for both the 5 and $8 \mathrm{~mm}$ displacements) and were followed by a holding phase in the displaced position lasting $1.5 \mathrm{sec}$. The intertrial intervals ranged from 1 to $5 \mathrm{sec}$. We began sampling (at a rate of 100 samples/sec) and storing the voltage across the potentiometers, together with the command input voltage to the torque motors, $100 \mathrm{msec}$ before the onset of the displacements. All subjects were repetitively tested on different days.

Because the goal of these experiments was to measure the forcedisplacement relation subserving hand posture, we attempted to prevent the occurrence of voluritary responses during bolh the movement and the holding phases of each trial. Subjects were asked, first, to focus on perceiving the direction of the displacement; second, to say aloud, "one-two"; and third, to move rapidly in the direction opposite to the imposed displacement. The rationale for this procedure was to increase the duration of the reaction time and to actually observe the occurrence of the voluntary response. In addition, during some of the sessions, we measured electromyographic (EMG) activity with surface electrodes from the biceps brachii, the triceps brachii (caput longus), the clavicular component of the pectoralis major, and the posterior deltoid to verify the absence of significant voluntary responses during the holding period ( 300 to $800 \mathrm{msec}$ ). The EMG signals were rectified and integrated (time constant, $50 \mathrm{~ms} \Theta \mathrm{c}$ ) before digital sampling and storage on disk.

Data processing. The voltage collected from the potentiometers and the voltage commands delivered to the servo amplifiers were transformed, by means of simple linear calibrations, into the joint angles of the mechanical apparatus and into the torques produced by the motors. The joint angles and torques were then converted into the cartesian coordinates of the hand and into the cartesian force at the same point by simple trigonometric transformations based on the kinematic equations of the manipulandum linkage.

The transformation from joint angles to handle position (in apparatus coordinates) is given by

$$
\begin{aligned}
& x^{\prime}=\ell_{1}{ }^{\prime} \cos \left(\theta_{1}{ }^{\prime}\right)+\ell_{2}{ }^{\prime} \cos \left(\theta_{1}{ }^{\prime}+\theta_{2}{ }^{\prime}\right) \\
& Y^{\prime}=\ell_{1}{ }^{\prime} \sin \left(\theta_{1}{ }^{\prime}\right)+\ell_{2}{ }^{\prime} \sin \left(\theta_{1}{ }^{\prime}+\theta_{2}{ }^{\prime}\right)
\end{aligned}
$$

where $\mathscr{C}_{1}{ }^{\prime}$ and $\ell_{2}{ }^{\prime}$ are the apparatus link lengths; $\theta_{1}{ }^{\prime}$ and $\theta_{2}{ }^{\prime}$ are, respectively, the apparatus "shoulder" and "elbow" angles; and $\left(x^{\prime}, y^{\prime}\right)$ are the coordinates of the handle. 
The transformation from torques to force is given by

$$
\begin{gathered}
F_{x}{ }^{\prime}=\left[\frac{\cos \left(\theta_{1}{ }^{\prime}+\theta_{2}{ }^{\prime}\right)}{\ell_{1}^{\prime}} T_{1}{ }^{\prime}-\frac{\cos \left(\theta_{1}{ }^{\prime}\right)}{\ell_{2}{ }^{\prime}} T_{2}{ }^{\prime}\right] \cdot\left[\sin \left(\theta_{2}{ }^{\prime}\right)\right]^{-1} \\
F_{y}{ }^{\prime}=\left[\frac{\sin \left(\theta_{1}^{\prime}+\theta_{2}{ }^{\prime}\right)}{\ell_{1}^{\prime}} T_{1}{ }^{\prime}-\frac{\sin \left(\theta_{1}^{\prime}\right)}{\ell_{2}^{\prime}} T_{2}{ }^{\prime}\right] \cdot\left[\sin \left(\theta_{2}{ }^{\prime}\right)\right]^{-1}
\end{gathered}
$$

where $T_{1}{ }^{\prime}$ and $T_{2}{ }^{\prime}$ are the torques generated by the two motors and $F_{x}{ }^{\prime}$ and $F_{y}{ }^{\prime}$ are the components of the corresponding force at the handle taken in the apparatus frame. Equation 2 was used to derive the handle coordinates in the subject's frame, while for the force we have

$$
\begin{aligned}
& F_{x}=-F_{x}{ }^{\prime} \\
& F_{y}=-F_{y}{ }^{\prime}
\end{aligned}
$$

when $F_{x}$ and $F_{y}$ are the force components in the subject's frame. Once the coordinates of the hand had been derived with respect to the subject's shoulder, the tangential velocity, given by

$$
v=\left(\dot{x}^{2}+\dot{y}^{2}\right)^{1 / 2}
$$

was computed. The time derivatives $\dot{x}$ and $\dot{y}$ were obtained by local polynomial fitting.

Typical data obtained in this way from a single displacement trial are shown in Figure 2. The initial motion is clearly indicated by the initial change in force and by a bell-shaped profile in the velocity trace. During the holding phase the velocity of the hand and, consequently, the power exchanged between the subject and the apparatus, are both almost zero for several hundred milliseconds. During this period we observed a significant and measurable force exerted by the subject on the handle. Since the hand was at rest, this force has no viscous or inertial components and represents a static restoring force that was driving the hand toward the original equilibrium position. After this holding phase we observed a sudden increase both in force and in velocity that corresponded to the onset of the voluntary response produced according to the instructions given to our subjects. The computer

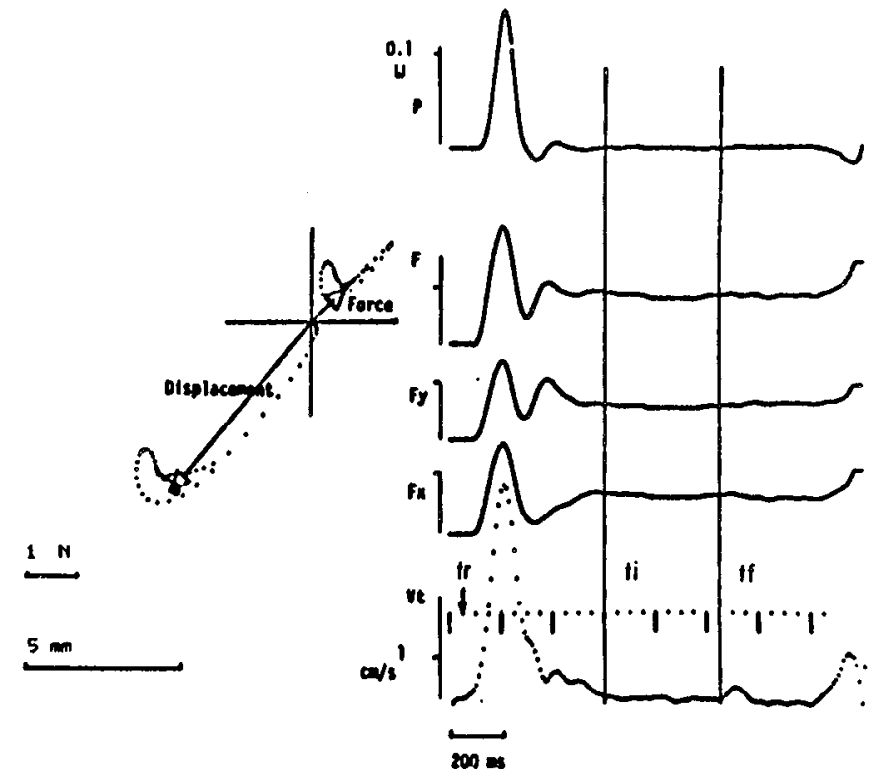

Figure 2. Typical force and position signal. The data collected during a servoed displacement are converted into cartesian force and position variables. The traces to the right are, from bottom to top, the hand tangential velocity (centimeters per second), the two components of the force at the handle obtained from the proportional control signal (newtons $(N)$ ), its magnitude, and the instantaneous mechanical power (watts). During the transient phase of the displacement, the force traces express only a portion of the actual force at the handle, since the velocity feedback component is neglected here. On the lett side, the position and force trajectories are plotted (dotted lines) in the cartesian plane. On the velocity trace, the arrow at $t_{f}$ indicates the beginning of the servoed displacement. Between $t_{i}$ and $t_{f}$, the hand has been displaced and the velocity is lower than a preselected threshold (in this case, $0.3 \mathrm{~cm} / \mathrm{sec}$ ). The samples included in this interval are averaged in order to compute a force and a displacement vector. These vectors are collected over several consecutive trials for the computation of hand stiffness. program automatically scanned the data for those portions of the trial characterized by zero velocity. The average vectors of restoring force and displacement were computed on the basis of the data collected during these portions of the trial (indicated by the arrows in Fig. 2).

For small displacements about an equilibrium position, the imposed displacements and the resulting forces are related by the following linear equations:

$$
\begin{aligned}
& F_{x}=-K_{x x} d x-K_{x y} d y \\
& F_{y}=-K_{y x} d x-K_{y y} d y
\end{aligned}
$$

where $F_{x}$ and $F_{y}$ are the components of the restoring force; $d x$ and $d y$ are the components of the imposed displacement; and the four coefficients $K_{x x_{1}}$ $K_{x y}, K_{y x}$, and $K_{y y}$ are the elements of the stiffness. ${ }^{3}$ The stiffness was estimated by using standard linear least squares regression procedures to determine these four coefficients. The square root of the variance associated with each coefficient was also computed using standard statistical methods (Kendall, 1961). In estimating the stiffness we did not impose any restriction on its elements; in particular, we did not require that it be symmetric. ${ }^{4}$

Stiffness representation. The linear force-displacement relation may be written in vector/matrix notation as

$$
\left[\begin{array}{l}
F_{x} \\
F_{y}
\end{array}\right]=\left[\begin{array}{ll}
K_{x x} & K_{x y} \\
K_{y x} & K_{y y}
\end{array}\right]\left[\begin{array}{l}
d x \\
d y
\end{array}\right] \quad \text { or } \quad \underline{F}=\underline{K} d \underline{\underline{x}}
$$

The stiffness, represented by the matrix, $\underline{K}$, was separated into a symmetric component, $\underline{K}_{s}$, and an antisymmetric component, $\underline{K}_{a}$, as follows:

$$
\begin{gathered}
\underline{K}_{s}=\left[\begin{array}{l}
K_{x x}\left(K_{x y}+K_{y x}\right) / 2 \\
\left(K_{y x}+K_{x y}\right) / 2 K_{y y}
\end{array}\right] \\
\underline{K}_{a}=\left[\begin{array}{l}
0\left(K_{x y}-K_{y x}\right) / 2 \\
\left(K_{y x}-K_{x y}\right) / 2
\end{array}\right]
\end{gathered}
$$

Note that $\underline{K}=\underline{K}_{s}+\underline{K}_{a}$

$$
\begin{aligned}
& { }^{3} \text { It is important to point out that the definition of stiffness does not imply } \\
& \text { a lincar forcc displaccmont relation. If } F=F(x, y) \text { is a differentiable nonlinear } \\
& \text { function of the position, it is possible to express the stiffness as a differential } \\
& \text { operator that relates small variations of force with small displacements, i.e., } \\
& \qquad d F_{x}=\left(\frac{\partial F_{x}}{\partial x}\right) \cdot d x+\left(\frac{\partial F_{x}}{\partial x}\right) \cdot d y=K_{x x} \cdot d x+K_{x y} \cdot d y \\
& \qquad d F_{y}=\left(\frac{\partial F_{x}}{\partial x}\right) \cdot d x+\left(\frac{\partial F_{y}}{\partial y}\right) \cdot d y=K_{y x} \cdot d x+K_{y y} \cdot d y
\end{aligned}
$$

These equations are strictly correct for infinitesimal displacements. We used small servoed displacements (less than $1 \mathrm{~cm}$ ) in order to approximate as much as possible a linear behavior, within the limits of the experimental resolution.

${ }^{4} \mathrm{~K}$ is said to be symmetric if

$$
K_{x y}=K_{y x}
$$

The physical meaning of this property is that if $K$ is symmetric, the force field $F(x, y)$ is conservative and a potential function $E_{\rho}(x, y)$ can be defined such that

$$
\underline{F}=(x, y)=-\operatorname{grad} E_{\rho}(x, y)
$$

In fact, a necessary and sufficient condition for $F(x, y)$ to be conservative is

$$
\frac{\partial F_{x}}{\partial y}=\frac{\partial F_{x}}{\partial x} \text { i.e., } K_{x y}=K_{y x}
$$

If, on the contrary,

$$
\frac{\partial F_{x}}{\partial x} \not \frac{\partial F_{y}}{\partial y}
$$

it is always possible to define the quantity

$$
\text { curl } \underline{E}(x, y)=\frac{\partial F_{y}}{\partial x}-\frac{\partial F_{x}}{\partial y}=K_{y x}-K_{x y}
$$

which is directly related to the total amount of mechanical work required to move the hand along a closed path. 


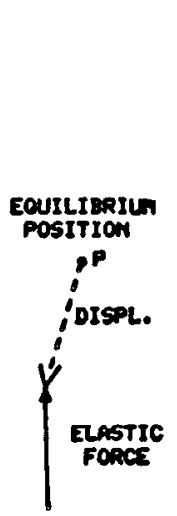

A

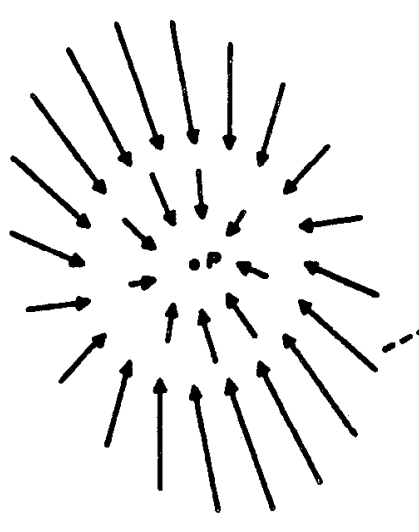

B

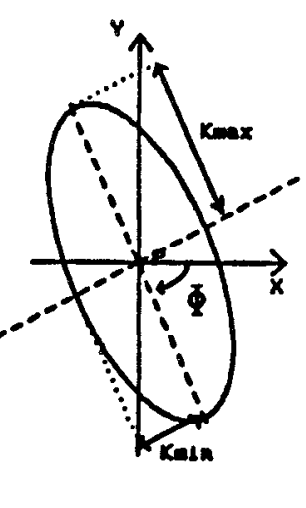

$c$
Figure 3. Stiffness representation. $A$, When the hand is displaced from its equilibrium position, an elastic restoring force is observed, which, in general is not co-linear with the displacement vector. $B$, Several displacements of variable amplitude and direction are plotted together with the restoring forces computed from a measured hand stiffness. $C$. The trajectory of the force vectors obtained by means of the previous procedure is an ellipse with the major and minor axes indicated, respectively, by $K_{\max }$ and $K_{\min }$. The angle, $\Phi$, between the major axis and the fixed $x$ axis is the stiffness orientation. The shape is given by the ratio $K_{\max } / K_{\min }$ and the size, or magnitude, is the area enclosed by the ellipse.
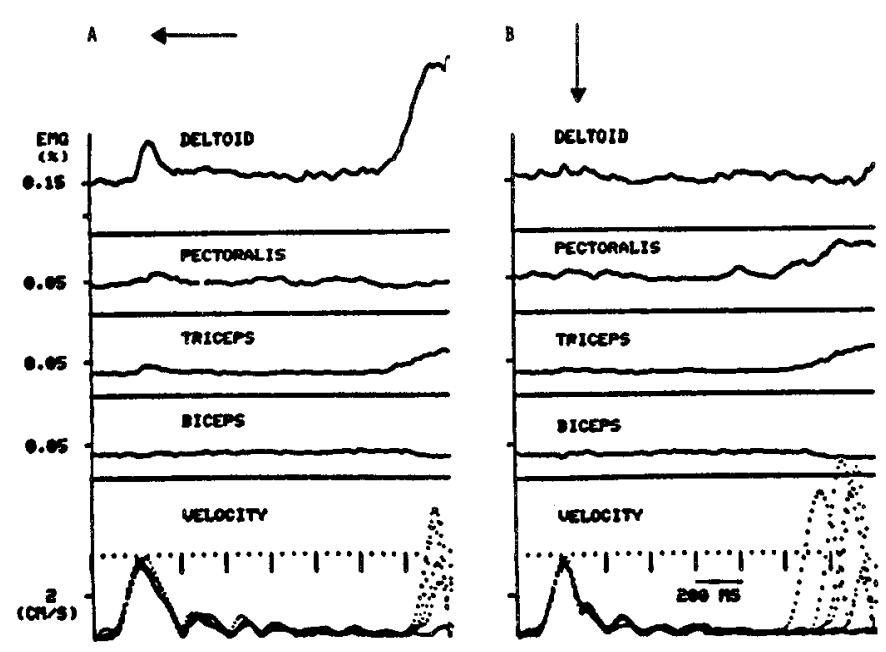

Figure 4. EMG activitics during scrvo displacements. The traces, from bottom to top, represent: hand velocity profiles obtained in individual servo displacements; and average EMG activities, over 12 trials, measured on biceps, triceps, pectoralis, and posterior deltoid (the EMGs are expressed as percentages of the activity recorded on each muscle during maximum isometric effort). The arrows at the top indicate the direction of the servo displacements: $A$, from right to left; $B$, toward the subject's shoulder, along the $y$ axis. The amplitude of displacement was always $8 \mathrm{~mm}$. All of the traces have been obtained from the same subject, in the same experimental session.

The antisymmetric component is characterized by a singlc parameter which is one-half of the curl of the vector force field generated by the hand under these experimental conditions. The symmetric component is characterized by the remaining three parameters. It may be represented geometrically by multiplying a hypothetical rotating input displacement of unit amplitude, e.g.,

$$
\begin{aligned}
& d x=\cos t \\
& d y=\sin t
\end{aligned} \quad 0<t<2 \pi
$$

by the symmetric stiffness matrix and plotting the corresponding output force vectors. They describe an ellipse that we refer to as the stiffness ellipse (Гig. 3).
The qualitative interpretation of the stiffness ellipse is straightforward: the major axis represents the direction along which the subject's arm is stiffest, whereas the minor axis is the direction of minimum stiffness. These two are the only directions in which the imposed displacement and the resulting force are perfectly co-linear, and these directions are referred to as the eigenvectors of the symmetric stiffness component. In any other direction the imposed displacement and the resulting force are not aligned. The stiffness in the directions of the major and minor axes are known as the eigenvalues of the symmetric stiffness component.

To facilitate the interpretation of the experimental data, the three parameters that characterize the symmetric stiffness component were expressed in terms of size, shape, and orientation of the ellipse. These quantities are easily seen on visual inspection. The size corresponds to the area of the ellipse proportional to the determinant of the symmetric stiffness, the shape is obtained as the ratio of the larger eigenvalue to the smaller, and the orientation of the ellipse is defined as the angle between the major axis (the principal eigenvector) and the $x$ axis. ${ }^{5}$

Verification of computational methods. In order to verify the data collection and analysis procedures and to quantify any random errors they introduced, we attached a set of linear mechanical springs to the handle of the manipulandum. By applying the program of servo-generated displacements to this spring arrangement, we computed its elastic field at its equilibrium position. Comparing the result with the actual stiffness of the system (determined by an independent measurement of the spring stiffness), we obtained an error margin of less than $5 \%$ on the estimate of the size, shape, and orientation of the elastic field.

\section{Results}

EMG activity during servoed displacements. The rectified and integrated EMG activities recorded from the biceps, the triceps, the pectoralis, and the deltoid are shown in Figure 4, together with the velocity of the hand during typical servoed displacements along the $x$ axis (Fig. 4A) and the $y$ axis (Fig. 4B). The EMG traces have been obtained from an average over 12 displacements produced during the same experimental session. During the displacements, the velocity traces have a characteristic bell-shaped profile with acceleration followed by deceleration and by minor oscillations of rapidly decreasing amplitude. Some of the EMG traces (e.g., posterior deltoid in Fig. 4A) show a dynamic response (stretch) starting about $50 \mathrm{msec}$ after the beginning of the mechanical transient and lasting as long as the displacement itself. Its magnitude depends upon the

${ }^{5}$ An alternative description of the symmetric component of the stiffness field can be given by means of isopotential energy curves (I logan, 1980 , $1984 b)$. In the proximity of the equilibrium position, these curves are ellipses defined by the equation

$K_{x x}\left(x-x_{0}\right)^{2}+K_{y y}\left(y-y_{0}\right)^{2}+2 K_{x y}\left(x-x_{0}\right)\left(y-y_{0}\right)$

$$
=2 t_{\rho}=\text { constant }
$$

If $K_{\min }$ and $K_{\max }$ are, respectively, the minimum and the maximum eigenvalues of the stiffness matrix, then the minimum and maximum axes $\left(r_{\min }\right.$ and $\left.r_{\max }\right)$ of the isopotential curve are given by

$$
\begin{aligned}
& r_{\max }=\left(\frac{2 E_{p}}{K_{\min }}\right)^{1 / 2} \\
& r_{\text {min }}=\left(\frac{2 E_{p}}{K_{\max }}\right)^{1 / 2}
\end{aligned}
$$

It follows that

$$
\frac{r_{\max }}{r_{\min }}=\left(\frac{K_{\max }}{K_{\min }}\right)^{1 / 2}
$$

i.e., that the shape of the isopotential ellipses is given by the square root of the stiffness shape. From this consideration it follows that, although a description in terms of potential energy seems more appropriate for theoretical considerations (e.g., potential energy is invariant with respect to coordinate changes), the stiffness ellipses provide more "sensitivity" to the difference in stiffness along different directions. 
direction of the servoed displacement, as can be seen by comparing the EMG traces in Figure 4, $A$ and $B$. When the hand comes to rest in the displaced position, the electromyograms (EMGs) return to levels which were approximately similar to those observed before the activation of the servo: a fact indicating that, during the holding period, there was no detectable sign indicating the presence of a voluntary response. On the basis of the average EMG during steadystate displacement, we cannot assess the amount of reflex activity contributing to the restoring force. We would like to re-emphasize here that the measurement of forces and displacements used to compute hand stiffness were all made during the period of time between the end of the dynamic response and the beginning of the voluntary reaction, i.e., at zero hand velocity.

Significance of the measuring procedure. The evaluation of the stiffness matrix is based upon a least squares regression of force onto displacement. How well does this procedure capture the information content of the real biological data? Figure 5 a represents, in a vector diagram, the force vectors as they were actually generated by the neuromuscular system in response to displacement from the position $P$ when a subject was holding the hand in the "reference" position. The displacement vectors are represented by the distance between $P$ and the tips of the arrows. Figure $5 b$ has been obtained after the stiffness matrix was computed from the displacement and force vectors shown in Figure $5 \mathrm{a}$. Using the measured displacements as an input to equation 14, we derived the force vectors shown in Figure $5 b$. These vectors represent the forces that would be generated by a system characterized exactly by the measured stiffness matrix, in response to the same displacements. By comparing Figure 5, $a$ and $b$, we conclude that our method was adequate to capture the most salient geometrical features of the force field associated with human arm posture. In particular, the matrix, $K$, allows us to predict the directions of maximum and minimum resistance to external disturbances, and makes it possible to predict, along any direction, the increase of the restoring force corresponding to increasing amplitude of displacement.

Predominance of spring-like behavior. Is the neuromuscular system spring-like? If so, the relation between displacement vectors and resulting force vectors must be without curl. That is, the measured stiffness for small displacements about an equilibrium position must be symmetrical. The measured stiffness matrices for

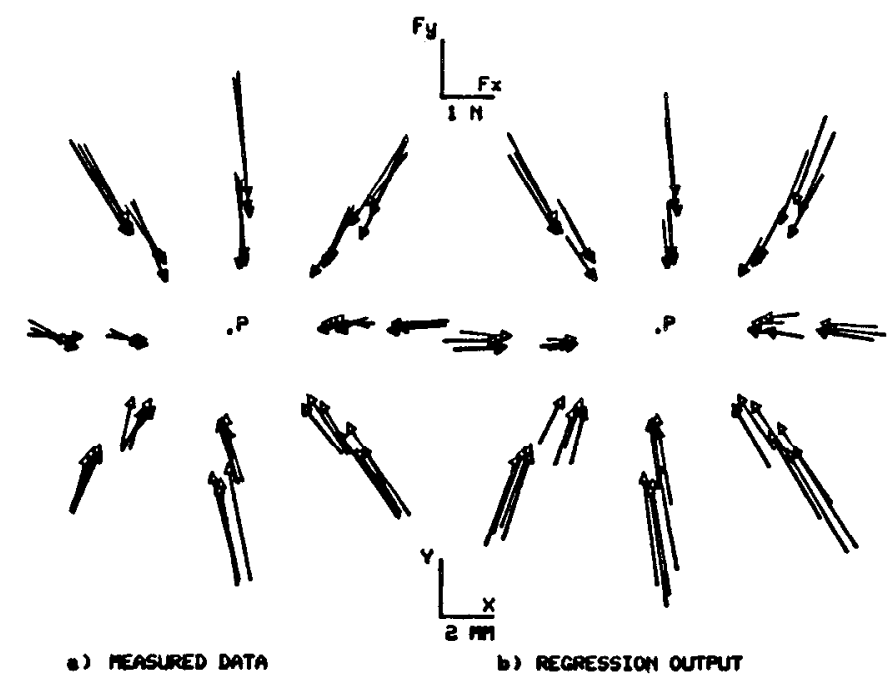

Figure 5. Estimate of the stiffness matrix. a, Vector diagram of the restoring forces measured from subject $A$ in the position "reference" (the diagram is obtained by the procedure illustrated in Fig. $3, A$ and $B$ ). $P$ is the equilibrium position of the hand before the displacements. $b$, Vector diagram of the restoring forces computed from the stiffness matrix resulting from the data in $a$. The forces were obtained by utilizing the actual displacements in the equation $\underline{I}=\underline{K} \cdot \underline{d x}$. two subjects maintaining a series of equilibrium postures of the hand at different locations in the work space are shown in Table I. The numerical values of the antisymmetric (curl) component of the stiffness are listed in the third column of Table II. The maximum and minimum eigenvalues of the symmetric stiffness, i.e., the maximum and minimum stiffness due to spring-like forces, are shown in the last two columns of Table II. It can be seen that the curl term, which represents any non-spring-like force, is in general small. We recall that, although the eigenvalues each contribute to a component of the restoring force, only one-half of the curl is affecting the individual components of this force. A representation of curl's magnitude relative to the entire field of restoring forces is shown in Figure 6 . Here the stiffness matrix has been used to derive the force vectors represented as arrows. The stiffness matrix measured from subject A in position "reference" (the numerical values are in Tables I and II) has been decomposed into its symmetric and antisymmetric components. The symmetric stiffness was used to generate the field of Figure $6 a$ while the antisymmetric one, which expresses the nonconservative stiffness, produced the field of Figure $6 \mathrm{~b}$. Comparing the two pictures makes it clear that the conservative component is largely predominant over the rotational one (curl).

A statistical estimate of the curl measured from our subjects in all tested work space locations is shown in Table III, together with the measured curl values for the mechanical springs placed in several orientations. The table also summarizes the results of a statistical $t$ test carried out to establish whether the observed curl is significantly different from zero. First, to determine how much of the measured curl was artifactual, we tested the hypothesis that the curl of the mechanical spring system is zero. Note that, because this mechanical system is purely elastic and contains no energy sources, any measured curl is artifactual and due to the measurement procedure. At high confidence levels $(p=0.01)$, the values should be considered non-zero when the springs are oriented at 0 and $60^{\circ}$. In the other three cases, the null hypothesis is accepted. Then, to determine whether the values of curl observed in the experimental subjects are significant, we performed a $t$ test comparing the curl measured from four experimental subjects with that measured from the spring apparatus. Since the data shown in Table III indicate a possible dependence of the measures on the stiffness orientation, the curl measured in the subjects was compared with that measured in the springs at similar orientations. The results are shown in Table IV. In summary, in two of our subjects ( $B$ and $C$ ), the hypothesis that the curl was zero (i.e., that the stiffness was spring-like) could not be rejected in aimost all work-space locations, whereas in two subjects ( $A$ and $D$ ) it should clearly be rejected. Given that the curl may be non-zero in some subjects, how large is it compared to the spring-like component of the steady-state behavior? To compare the magnitude of the spring-like and non-spring-like forces, we computed the ratio of the force due to the curl alone to the maximum and minimum force due to the spring-like stiffness. These ratios are shown as percentages in Table $V$ as $Z_{\min }$ and $Z_{\max }$.

The two numbers $Z_{\max }$ and $Z_{\min }$ compare the non-spring-like forces with the total forces for displacements of the end-point in the direction of least and greatest stiffness, respectively. However, they suffer the drawback that their values are quite sensitive to the coordinate frame in which the comparison is made. It is possible to make an alternative comparison that is independent of the coordinate frame in which the measurements are made and is, therefore, the least sensitive to errors introduced by our measurement apparatus. It is the square root of the ratio of the determinant of the antisymmetric stiffness to the determinant of the symmetric stiffness, or, alternatively, the geometric mean of $Z_{\max }$ and $Z_{\min }$, and it is shown as a percentage in Table $V$ as $Z_{\text {mean }}$.

Using $Z_{\text {mean }}$ as a measure, it can be seen that in all cases but one, the magnitude of the curl term is less than $14 \%$ of the magnitude of the spring-like forces. Even if we use $Z_{\max }$ as a worst-case measure of the magnitude of the curl, it can be seen that in all cases but three, the curl is responsible at most for less than $25 \%$ and in the 
TABLE I

Estimated values for the stiffness tensor in cartesian hand coordinates and in joint coordinates

The joint stiffness terms refer to the following equations:

$$
\begin{aligned}
& T_{1}=R_{11} \cdot d \theta_{1}+R_{12} \cdot d \theta_{2} \\
& T_{2}=R_{21} \cdot d \theta_{1}+R_{22} \cdot d \theta_{2}
\end{aligned}
$$

where $T_{1}$ and $d \theta_{1}$ represent the torque and the angulr displacement at the shoulder, and $T_{2}$ and $d \theta_{2}$ represent the torque and the angular displacement at

\begin{tabular}{|c|c|c|c|c|c|}
\hline Subject & $\begin{array}{l}\text { Hand } \\
\text { Position }\end{array}$ & \multicolumn{2}{|c|}{$\begin{array}{l}\text { Hand Stiffness } \\
(\mathrm{Nw} / \mathrm{m})^{2}\end{array}$} & $\begin{array}{r}\text { Join } \\
\text { (Nv } \\
R 11 \\
R 21 \\
\end{array}$ & $\begin{array}{l}\text { ness } \\
\text { rad) } \\
R 12 \\
R 22 \\
\end{array}$ \\
\hline \multirow[t]{8}{*}{$A$} & Reference & $\begin{array}{c}-173 \pm 7 \\
27.4 \pm \\
14\end{array}$ & $\begin{array}{l}72.8 \pm 8 \\
-387 \pm \\
17\end{array}$ & $\begin{array}{l}-24.1 \pm 1.5 \\
-13.4 \pm 2.4\end{array}$ & $\begin{array}{r}-8.5 \pm 1.9 \\
-36.0 \pm 3.0\end{array}$ \\
\hline & Distal & $\begin{array}{c}-114 \pm 5 \\
24.1 \pm \\
20\end{array}$ & $\begin{array}{l}71.8 \pm 8 \\
-641 \pm \\
33\end{array}$ & $\begin{array}{l}-29.9 \pm 2.1 \\
-18.8 \pm 3.6\end{array}$ & $\begin{array}{l}-13.3 \pm 2.3 \\
-39.1 \pm 4.0\end{array}$ \\
\hline & Proximal & $-251 \pm 9$ & $\begin{array}{l}68.3 \pm \\
10\end{array}$ & $-19.0 \pm 1.1$ & $-7.0 \pm 1.6$ \\
\hline & & $\begin{array}{l}44.8 \pm \\
17\end{array}$ & $\begin{array}{c}-354 \pm \\
19\end{array}$ & $-9.0 \pm 2.2$ & $-38.0 \pm 3.1$ \\
\hline & Right & $\begin{array}{c}-248 \pm \\
18\end{array}$ & $\begin{array}{c}-158 \pm \\
21\end{array}$ & $-29.0 \pm 8.9$ & $-9.9 \pm 5.0$ \\
\hline & & $\begin{array}{c}-207 \pm \\
20\end{array}$ & $\begin{array}{c}-409 \pm \\
24\end{array}$ & $-15.5 \pm 5.0$ & $-36.8 \pm 2.0$ \\
\hline & Left & $\begin{array}{c}-450 \pm \\
18\end{array}$ & $\begin{array}{l}349 \pm \\
17\end{array}$ & $-40.4 \pm 11.6$ & $-15.7 \pm 6.0$ \\
\hline & & $\begin{array}{l}265 \pm \\
22\end{array}$ & $\begin{array}{c}-436 \pm \\
21 \\
\end{array}$ & $-25.0 \pm 6.5$ & $-41.3 \pm 5.4$ \\
\hline \multirow[t]{7}{*}{$\mathrm{D}$} & Reference & $\begin{array}{r}-192 \pm 15 \\
62.8 \pm 17\end{array}$ & $\begin{array}{r}115 \pm 17 \\
-307 \pm 20\end{array}$ & $\begin{array}{r}-25.1 \pm 2.8 \\
-7.5 \pm 3.1\end{array}$ & $\begin{array}{r}-2.3 \pm 3.1 \\
-21.5 \pm 3.4\end{array}$ \\
\hline & Distal & $-125 \pm 11$ & $112 \pm 14$ & $-30.2 \pm 4.0$ & $-6.5 \pm 3.7$ \\
\hline & & $66.3 \pm 19$ & $\begin{array}{l}-436 \quad \pm \\
4.0\end{array}$ & $-11.2 \pm 4.0$ & $-18.3 \pm 3.7$ \\
\hline & Proximal & $\begin{array}{r}-294 \pm 13 \\
44.2 \pm 15\end{array}$ & $\begin{array}{r}59.6 \pm 12 \\
-291 \pm 14\end{array}$ & $\begin{array}{r}-16.4 \pm 1.1 \\
-4.3 \pm 1.4\end{array}$ & $\begin{array}{r}-3.3 \pm 1.4 \\
-28.3 \pm 1.9\end{array}$ \\
\hline & Right & $\begin{array}{l}-138 \pm 8 \\
-72.2 \pm 10\end{array}$ & $\begin{array}{c}-68.1 \pm 9 \\
-259 \pm 12\end{array}$ & $\begin{array}{r}-25.3 \pm 4.2 \\
-8.5 \pm 2.1\end{array}$ & $\begin{array}{r}-8.1 \pm 2.2 \\
-16.3 \pm 1.1\end{array}$ \\
\hline & Left & $-169 \pm 17$ & $123 \pm 17$ & $-17.1 \pm 9.9$ & $-8.5 \pm 4.8$ \\
\hline & & $109 \pm 15$ & $-175 \pm 15$ & $-9.9 \pm 4.5$ & $-13.4 \pm 2.2$ \\
\hline
\end{tabular}
the elbow.

${ }^{a} \mathrm{Nw} / \mathrm{m}$, newtons per meter.

${ }^{b} \mathrm{Nw} \cdot \mathrm{m} / \mathrm{rad}$, newton $\cdot$ meters per rad.

majority of the cases for less than $10 \%$ of the restoring forces generated by the symmetric stiffness component. In summary, in some subjects, the non-spring-like behavior is not statistically significant and, in almost all cases, it is small compared to the spring like behavior.

Regularities in spring-like behavior. The typical spring-like behavior of four subjects performing the postural task is shown in Figure 7. The stiffness ellipses measured at five work space positions are shown along with a schematic display of the corresponding arm configurations. A remarkable feature of these data is the similarity of the stiffness ellipses for different subjects. In particular, at any given location, the shape and the orientation of the ellipses do not change substantially from one subject to another. In contrast, the stiffness magnitude varied considerably.

The results obtained from the same subject on different days are shown in Figure 8A. The stiffness ellipses have been computed for individual runs, i.e., on the basis of about 30 servoed displacements, during which the subject was asked to maintain posture in four work space positions. The figure shows the variation in size, shape, and orientation of the stiffness measured over a time interval of several months. The orientation and the shape of the ellipses obtained at a given position are almost constant, whereas the magnitude varies considerably. To separate the variability in magnitude from that in shape and orientation, the stiffness ellipses have been normalized to enclose the same area in Figure $8 B$. The elements of each stiffness matrix were divided by the square root of its determinant. Comparing the normalized ellipses to the original ones, it is evident that most of the variability has been eliminated, as would be expected if the shape and the orientation of the ellipses remain constant.

Patterns of postural stiffness. Table VI shows the observed values of the three parameters of the symmetric component of the measured stiffness, its size, shape, and orientation. Several observations are in order here. First, at each work space location, the postural behavior is anisotropic; it has a strong directional character. There is a substantial difference between the major and the minor axes of the ellipses, i.e., between the minimum and the maximum stiffness. Sccond, the size, the shapc, and the orientation of the stiffncss ellipse all change with the position of the hand in the work space. It follows that end-point stiffness in work space coordinates is not an invariant quantity. However, in all subjects, the variations of shape and orientation follow a regular and repeatable pattern (Fig. 7): along the proximal-distal direction there is a predominant change in the ellipse shape, with a less pronounced change in orientation. The shape parameters indicate that when the hand was in the "reference" position, the maximum stiffness was more than double the minimum 
TABLE \|

Antisymmetric and symmetric components of the stiffness

$K_{\max }$ and $K_{\min }$ are, respectively, the maximum and the minimum eigenvalues of the symmetric (spring-like) stiffness. The curl is given by the difference between the off-diagonal terms in the antisymmetric stiffness. In order to estimate the relative contribution of spring-like and non-spring-like terms to the restoring force field, the eigenvalues should be compared with one-half of the curl.

\begin{tabular}{clccc}
\hline Subject & Position & Curl & $K_{\max }$ & $K_{\min }$ \\
\hline \multirow{2}{*}{ A } & Reference & $-45.4 \pm 22.7$ & $-398.5 \pm 21.7$ & $-162.3 \pm 12.4$ \\
& Distal & $-47.7 \pm 27.8$ & $-645.6 \pm 35.3$ & $-109.4 \pm 7.5$ \\
& Proximal & $-23.5 \pm 26.8$ & $-378.9 \pm 26.9$ & $-226.3 \pm 20.6$ \\
& Right & $-48.5 \pm 41.4$ & $-527.9 \pm 41.3$ & $-128.7 \pm 38.6$ \\
& Left & $-84.3 \pm 39.1$ & $-750.2 \pm 39.1$ & $-136.2 \pm 39.1$ \\
$D$ & Reference & $-51.9 \pm 34.4$ & $-355.3 \pm 33.3$ & $-143.6 \pm 30.4$ \\
& Distal & $-45.9 \pm 35.2$ & $-459.5 \pm 35.2$ & $-100.9 \pm 21.1$ \\
& Proximał & $-14.5 \pm 27.7$ & $-343.5 \pm 27.6$ & $-240.6 \pm 27.7$ \\
& Right & $-4.0 \pm 19.7$ & $-291.2 \pm 18.8$ & $-105.6 \pm 16.2$ \\
& Left & $-14.1 \pm 32.5$ & $-287.8 \pm 32.5$ & $-55.9 \pm 32.5$ \\
\hline
\end{tabular}

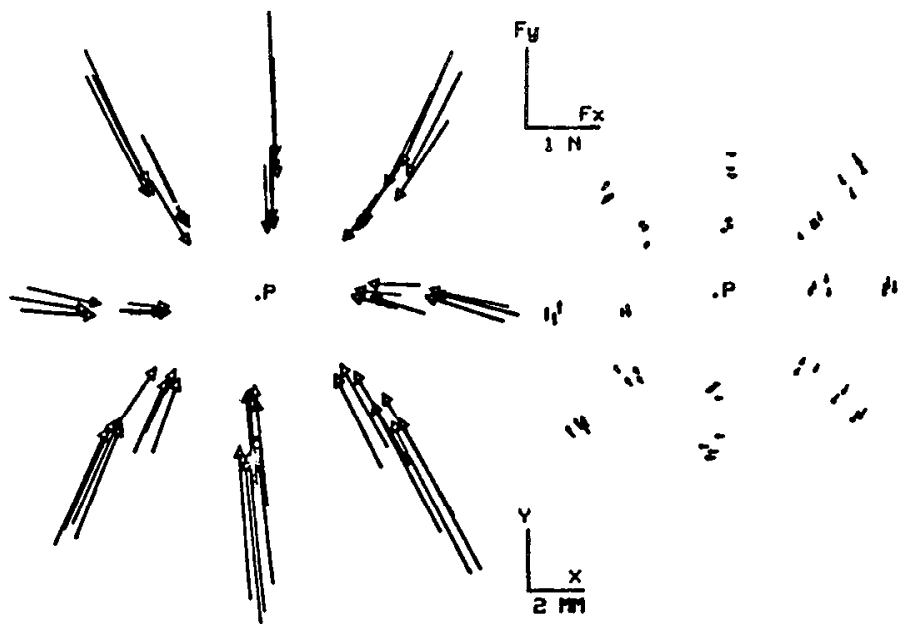

$\because$ conseruative component

b) ROTATIONAL CONPONENT

Figure 6. Conservative and rotational stiffness: their relative contribution to the field. The matrix used in Figure $5 b$ has been separated into asymmetric (conservative (a)) and an antisymmetric (rotational $(b)$ ) component to generate the two-vector diagrams.

stiffness. This anisotropy increased by about a factor of two as the hand moved to the "distal" position. A slight, although systematic, clockwise rotation of the ellipse was observed when the hand posture changed from proximal to distal.

In contrast, position changes along the right-left direction resulted in a substantial rotation of the stiffness ellipse, with a less pronounced change in its shape. Whereas the stiffness orientation changed by only about $15^{\circ}$ between the proximal and distal positions, a rotation of more than $65^{\circ}$ was observed between the right and the left positions, the major axis of the ellipse being approximately oriented toward the subject's shoulder.

Although the stiffness shape and orientation showed regular patterns of change, we detected no systematic trend in the changes in the stiffness magnitude as the arm posture changed in the work space. Furthermore, the stiffness magnitude at a given posture changed over time and among subjects, whereas the shape and orientation did not. These observations suggest that a coordinated synergistic change in the tonic activity of the arm muscles took place, since an arbitrary uncoordinated change in muscle activation should result in a substantial change of shape and orientation as well as magnitude. However, it must be remembered that the kinematics of the arm have a profound effect on the end-point stiffncss produced by any given level of muscle activity. An alter native hypothesis is that the arm stiffness is regulated or invariant in some coordinate frame other than that of the end-point, e.g., joint coordinates. The observed changes in shape and orientation with position in the work space would then be solely due to the geometric effects of musculoskeletal anatomy.

Computer simulation of constant joint stiffness. Figure 9 shows the results of a computer simulation in which the joint stiffness is kept constant while the hand is positioned in different locations, including the five used in the experiments. We computed the joint stiffness corresponding to the measured end-point stiffness in the "reference" positon and used this as the assumed constant joint stiffness. Then at each of the other positions we computed the endpoint stittness corresponding to this constant joint stiffness and drew the corresponding ellipses. Any change in end-point stiffness resulting from this procedure is solely due to geometric effects. Clearly, the changes in ellipse configuration shown in Figure 9 are qualitatively similar to those measured in our subjects. To an extent, this result is inevitable because of the dominant influence of limb kinematics. For example, as the elbow approaches its maximum extension, the hand stiffness is expected to increase along the radial line connecting the hand to the shoulder. In fact, when the elbow is fully extended, the arm can no longer move in the radial direction and, consequently, the hand stiffness will become infinite in this direction no matter what the joint stiffness is. However, there are important quantitative differences between Figure 7 (experimental subjects) and Figure 9 (simulations) that should not be underestimated. For example, the variations in the actual stiffness ellipses as the hand position changes are not as pronounced as the simulated ones. This is particularly evident in the proximal position, where the simulated ellipse is almost completely oriented along the $x$ axis, whereas the measured ellipse has changed little from the reference position.

Are the observed patterns of postural stiffness functionally significant? The total postural response of the limb to disturbances is due to the complex interplay of several aspects of its dynamic behavior, e.g., its stiffness, viscosity, and inertia. One possibility we have examined is that the observed changes in stiffness are coordinated with changes in these other aspects of postural dynamics. We investigated this idea by estimating the apparent end-point inertia of the limb and comparing it to the stiffness. The end-point inertia tensor was computed by modeling the subject's arm as a kinematic chain of two rigid links (forearm and upper arm), each with a uniform mass distribution (total masses: forearm, $1.30 \mathrm{~kg}$; upper arm, 2.52 $\mathrm{kg}$ ). Like the stiffness, the inertial behavior can be represented by an ellipse. Its major axis represents the direction of greatest mass; its minor axis represents the direction of least apparent mass. The solid ellipses in Figure 10 show how the inertia changes across the work space. Note that the direction of maximum inertia is almost parallel to the outer link. The dashed ellipses in Figure 10 show the stiffness ellipse measured from one of our subjects. It is remarkable that the angle between the major axes of the two ellipses shows relatively little variation, remaining within a range of 20 to $40^{\circ}$. The meaning of this constancy for motor coordination requires further investigations.

TABLE III

Significance of the curl measured in the calibration springs

\begin{tabular}{rccccc} 
Orientation & Curl \pm SD & Samples & $t$ & $p=0.05^{\circ}$ & $p=0.01^{\circ}$ \\
\hline $0^{\circ}$ & $8.20 \pm 7.65$ & 60 & 8.301 & $\mathrm{R}$ & $\mathrm{R}$ \\
$30^{\circ}$ & $3.87 \pm 13.99$ & 30 & 1.513 & $\mathrm{~A}$ & $\mathrm{~A}$ \\
$60^{\circ}$ & $-23.13 \pm 13.85$ & 30 & -9.147 & $\mathrm{R}$ & $\mathrm{H}$ \\
$90^{\circ}$ & $-2.99 \pm 10.58$ & 30 & -1.546 & $\mathrm{~A}$ & $\mathrm{~A}$ \\
$120^{\circ}$ & $4.63+9.49$ & 30 & 2.669 & $\mathrm{R}$ & $\mathrm{A}$ \\
\hline
\end{tabular}

${ }^{a}$ Null hypothesis: curl $=0$. A, hypothesis accepted; $R$, hypothesis rejected. 
TABLE IV

Tesi of the difference between the curl measured in the subjects and the curl measured in the spring

\begin{tabular}{|c|c|c|c|c|c|c|c|}
\hline Subject & $\begin{array}{l}\text { Hand } \\
\text { rosition }\end{array}$ & $\begin{array}{l}\text { Stiffness } \\
\text { Orientation }\end{array}$ & $\begin{array}{c}\text { Spring } \\
\text { Orientation }\end{array}$ & $\begin{array}{l}\text { Degrees } \\
\text { of } \\
\text { Freedom }\end{array}$ & $t$ & $\rho=0.05^{a}$ & $p=0.01^{a}$ \\
\hline \multirow[t]{4}{*}{ A } & Reference & 102.6 & 90 & 86 & -9.678 & $\mathrm{R}$ & $\mathrm{R}$ \\
\hline & Proximal & 113.9 & 120 & 87 & -5.547 & $\mathrm{R}$ & $\mathrm{R}$ \\
\hline & Right & 56.9 & 60 & 85 & -3.256 & $\mathrm{R}$ & $\mathrm{R}$ \\
\hline & Left & 135.7 & 120 & 85 & -12.236 & $\mathrm{R}$ & $\mathrm{R}$ \\
\hline \multirow{4}{*}{$\mathrm{B}$} & Proximal & 120.6 & 120 & 71 & 2.596 & $R$ & A \\
\hline & $\mathrm{RIIPR}^{b}$ & 80.7 & 90 & 67 & 0.661 & A & A \\
\hline & RI-DI. ${ }^{c}$ & 64.0 & 60 & 75 & -0.864 & $A$ & $A$ \\
\hline & Left & 126.8 & 120 & 81 & -6.634 & $R$ & $\mathrm{R}$ \\
\hline \multirow[t]{2}{*}{ C } & Reference & 104.9 & 90 & 82 & -0.678 & A & $A$ \\
\hline & Distal & 103.4 & 90 & 83 & 1.968 & $A$ & $A$ \\
\hline \multirow{4}{*}{ D } & Distal & 104.9 & 90 & 86 & -6.507 & $\mathrm{R}$ & $\mathrm{R}$ \\
\hline & Proximal & 135.9 & 120 & 86 & -3.652 & $\mathrm{R}$ & $\mathrm{R}$ \\
\hline & Right & 65.5 & 60 & 86 & 4.723 & $\mathrm{R}$ & $\mathrm{R}$ \\
\hline & Left & 134.4 & 120 & 85 & -3.083 & $\mathrm{R}$ & $\mathrm{R}$ \\
\hline
\end{tabular}

${ }^{a}$ Null hypothesis: curl (subjects) = curl (springs). A, hypothesis accepted; R, hypothesis rejected.

${ }^{b} \mathrm{RI}-\mathrm{PR}$, right proximal.

${ }^{c}$ Ri-DI, right distal.

TABLE V

Three measures of the relative magnitude of non-spring-like behavior ${ }^{2}$

\begin{tabular}{|c|c|c|c|c|}
\hline Subject & Position & $Z_{\min }$ & $Z_{\max }$ & $Z_{\text {mean }}$ \\
\hline \multirow[t]{5}{*}{ A } & Reference & 5.7 & 14.0 & 8.9 \\
\hline & Distal & 3.7 & 21.8 & 9.0 \\
\hline & Proximal & 3.1 & 5.2 & 4.0 \\
\hline & Right & 4.6 & 18.9 & 9.3 \\
\hline & Left & 5.6 & 31.0 & 13.2 \\
\hline \multirow[t]{6}{*}{ B } & Reference & 2.9 & 6.2 & 4.2 \\
\hline & Distal & 1.4 & 5.7 & 2.9 \\
\hline & Proximal & 3.4 & 5.7 & 4.4 \\
\hline & Right-proximal & 1.2 & 2.8 & 1.8 \\
\hline & Right-distal & 3.2 & 37.0 & 10.8 \\
\hline & Left & 5.8 & 76.5 & 20.9 \\
\hline \multirow[t]{5}{*}{ C } & Reference & 2.1 & 7.1 & 3.9 \\
\hline & Distal & 1.0 & 5.5 & 2.3 \\
\hline & Proximal & 3.6 & 8.7 & 5.6 \\
\hline & Right & 1.4 & 5.2 & 2.7 \\
\hline & Left & 0.2 & 0.9 & 0.4 \\
\hline \multirow[t]{5}{*}{ D } & Reference & 7.3 & 18.1 & 11.5 \\
\hline & Distal & 5.0 & 22.7 & 10.7 \\
\hline & Proximal & 2.1 & 3.0 & 2.5 \\
\hline & Right & 0.7 & 1.9 & 1.2 \\
\hline & Left & 2.5 & 12.6 & 5.6 \\
\hline Spring & $0^{\circ}$ & 0.4 & 0.9 & 0.6 \\
\hline \multirow[t]{4}{*}{ system } & $30^{\circ}$ & 0.2 & 0.4 & 0.3 \\
\hline & $60^{\circ}$ & 1.2 & 2.6 & 1.7 \\
\hline & $90^{\circ}$ & 0.1 & 0.4 & 0.2 \\
\hline & $120^{\circ}$ & 0.2 & 0.5 & 0.4 \\
\hline
\end{tabular}

${ }^{2}$ See the text for details.

Adaptive changes in postural stiffness. The ability to modulate the postural stiffness of the arm is one of the ways in which the CNS adapts its behavior to changing environmental conditions (Hogan, 1985). To investigate adaptive changes in stiffness, we asked subjects to maintain the hand at rest against an external destabilizing force. A schematic description of the procedure employed here is given in Figure 11. A force, of fixed direction and of sinusoidally varying amplitude, is imposed on the subject's hand by the torque motors. The task is to maintain the hand at rest. After about $2 \mathrm{sec}$, this force is removed and a servo displacement is applied (with a latency of about $1 \mathrm{sec}$ from the end of the oscillations) to measure the vectors used in the estimate of the stiffness. The process is then repeated 30 times in each experimental run. Three types of disturbances were tested: a rotating force of constant amplitude (frequency: 5 cycles/sec; amplitude: $8 \mathrm{~N}$ ), a force of slowly varying amplitude (frequency: 1 cycle/sec; amplitude; $10 \mathrm{~N}$ ) directed along the $x$ axis, and a force (same frequency and amplitude) directed along the $y$ axis. After the disturbance had been applied in brief pulses (30/ session) of 2 sec duralion, the stiffness of the hand was measured. It is important to note that, because our method of measuring stiffness required the arm to be in a steady-state posture, stiffness was measured about $2 \mathrm{sec}$ after the end of each pulse disturbance. As a result, the ellipses obtained in this experiment do not describe the stiffness during the compensation for the disturbance but are related to its aftereffects, e.g., a bias induced in the posture by a repetitive and predictable disturbance.

The data shown in Figure 12 were obtained in three work-space positions: reference, right, and left. It is quite clear that the principal effect induced by all of the disturbances was a global increase in the magnitude of stiffness. The changes in shape and orientation were minimal compared with variations in size. Hence, the strategy apparently adopted by our subjects was not to increase the stiffness selectively along the direction of the predictable disturbance, but to makc the wholc arm stiffer, presumably at the expense of a greater metabolic energy expenditure. We are, at present, investigating whether other behavioral paradigms may be capable of eliciting a change in orientation and/or shape.

Effects of changing arm configuration. As described previously, the stiffness of the hand is strongly influenced by the configuration of the musculoskeletal system. Because of the kinematic redundancy of the musculoskeletal system, the stiffness at the hand depends not only on the position of the hand in the work space, but also on the orientation of the arm supporting the hand. To investigate 

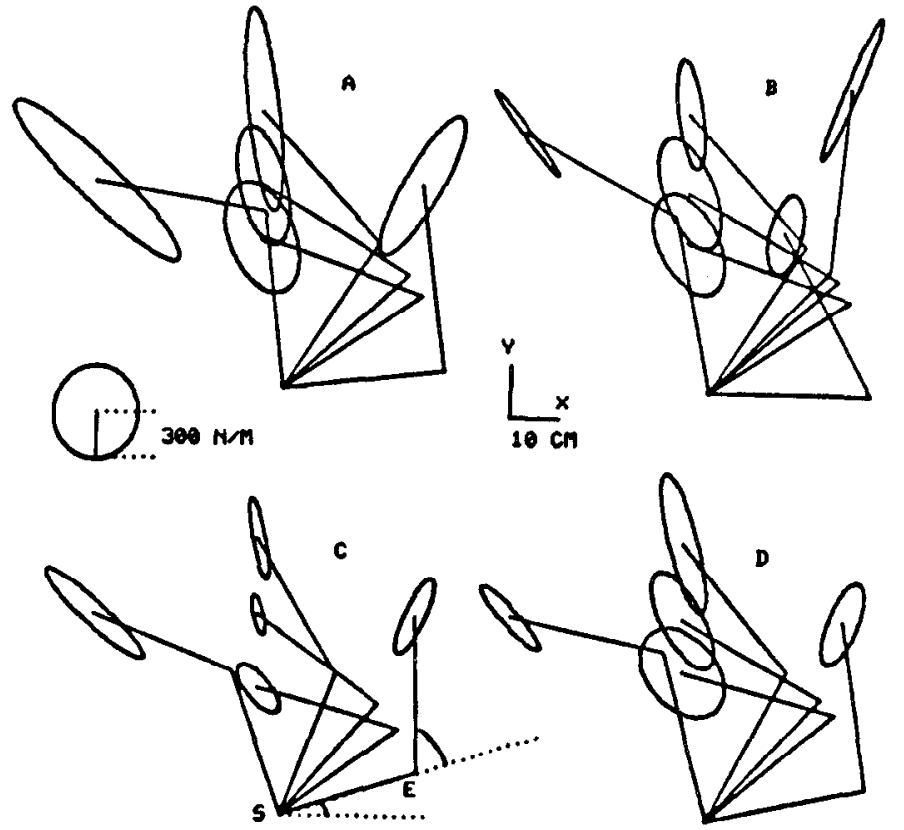

Figure 7. Stiffness ellipses obtained from four subjects during the postural task. Each ellipse has been derived by regression on about 60 force and displacement vectors. The upper arm and the forearm are indicated schematically by two line segments and the ellipses are placed on the hand. Subjects $A, C$, and $D$ were keeping posture in the five standard positions described in the text. Subject $B$ was requested to maintain the arm in two different positions on the right side (right-proximal: $x=15 \mathrm{~cm}, y=29.5 \mathrm{~cm}$; right-distal: $x=28 \mathrm{~cm}, y=57 \mathrm{~cm}$ ) and in a more distal position on the left $(x=-38 \mathrm{~cm}, y=48.5 \mathrm{~cm})$. The calibration for the stiffness is provided by the circle to the left which represents an isotropic hand stiffness of $300 \mathrm{~N} /$ $\mathrm{m}$.

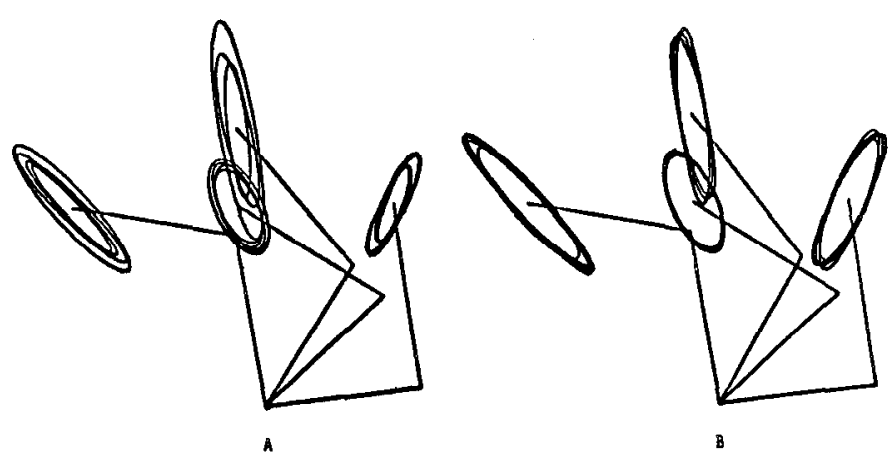

Figure 8. Stiffness variability. A, Stiffness ellipses measured in subject $A$ on four different days over a period of 6 months. $B$, The same ellipses normalized in such a way as to cover the same area.

TABLE VI

Geometrical parameters of the stiffness ellipses obtained from subjects $A$

\begin{tabular}{clrrr}
\multicolumn{5}{c}{ and $D^{\mathbf{a}}$} \\
\hline \multirow{2}{*}{ Subject } & Position & \multicolumn{1}{c}{$\begin{array}{c}\text { Size } \\
(\mathrm{N} / \mathrm{cm})^{2}\end{array}$} & $\begin{array}{c}\text { Orientation } \\
\text { (Degrees) }\end{array}$ & \multicolumn{1}{c}{ Shape } \\
\hline \multirow{2}{*}{$\mathrm{A}$} & Reference & $20.3 \pm 2.2$ & $102.6 \pm 3.8$ & $2.46 \pm 0.29$ \\
& Distal & $22.2 \pm 2.6$ & $95.2 \pm 1.8$ & $5.90 \pm 0.70$ \\
& Proximal & $26.9 \pm 3.0$ & $113.9 \pm 7.2$ & $1.68 \pm 0.21$ \\
& Right & $21.3 \pm 6.5$ & $56.9 \pm 4.0$ & $4.10 \pm 1.20$ \\
& Left & $32.0 \pm 9.2$ & $135.7 \pm 1.9$ & $5.51 \pm 1.58$ \\
$\mathrm{D}$ & Reference & $16.0 \pm 3.6$ & $118.5 \pm 6.5$ & $2.47 \pm 0.55$ \\
& Distal & $14.6 \pm 3.6$ & $104.9 \pm 4.0$ & $4.56 \pm 1.12$ \\
& Proximal & $26.0 \pm 3.0$ & $135.9 \pm 7.9$ & $1.43 \pm 0.16$ \\
& Right & $9.7 \pm 1.6$ & $65.5 \pm 4.3$ & $2.76 \pm 0.47$ \\
& Left & $5.1 \pm 2.9$ & $134.4 \pm 4.1$ & $5.15 \pm 2.99$ \\
\hline
\end{tabular}

${ }^{a}$ These data were computed from the matrices reported in Table I.

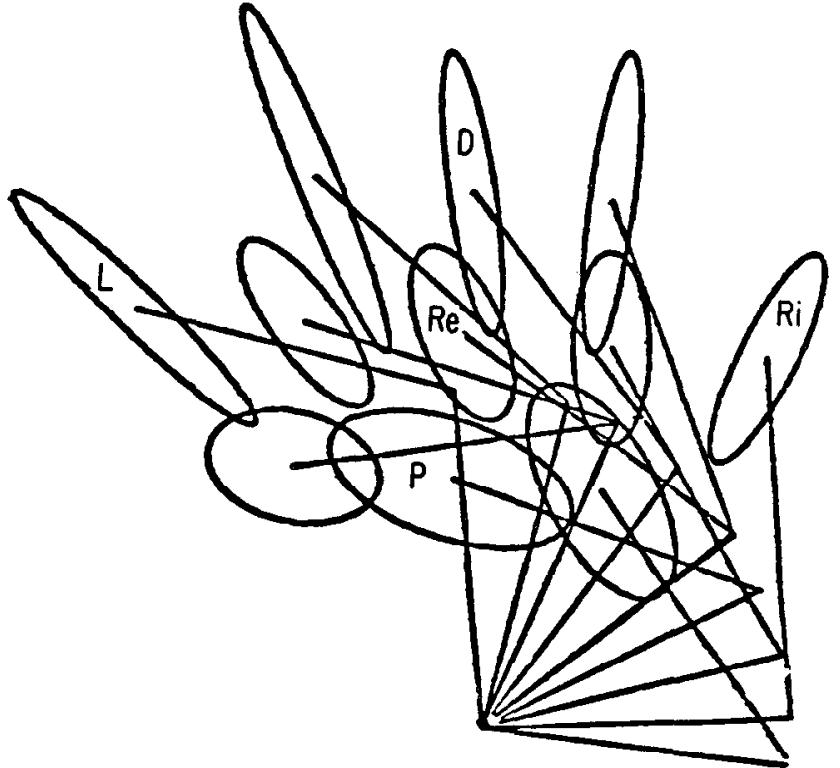

Figure 9. Simulation of constant joint stiffness. The joint stiffness tensor has been computed from the cartesian stiffness of subject $A$ during hand posture in position "reference" $(R e)$ (see Table I and Fig. 7). With all the joint stiffness terms kept constant, the model of the arm has been displaced to 11 work space positions and the corresponding cartesian stiffnesses have been computed. The five positions where the stiffness was measured have been included: $D$, distal; $P$, proximal; $R i$, right; $L$, left).

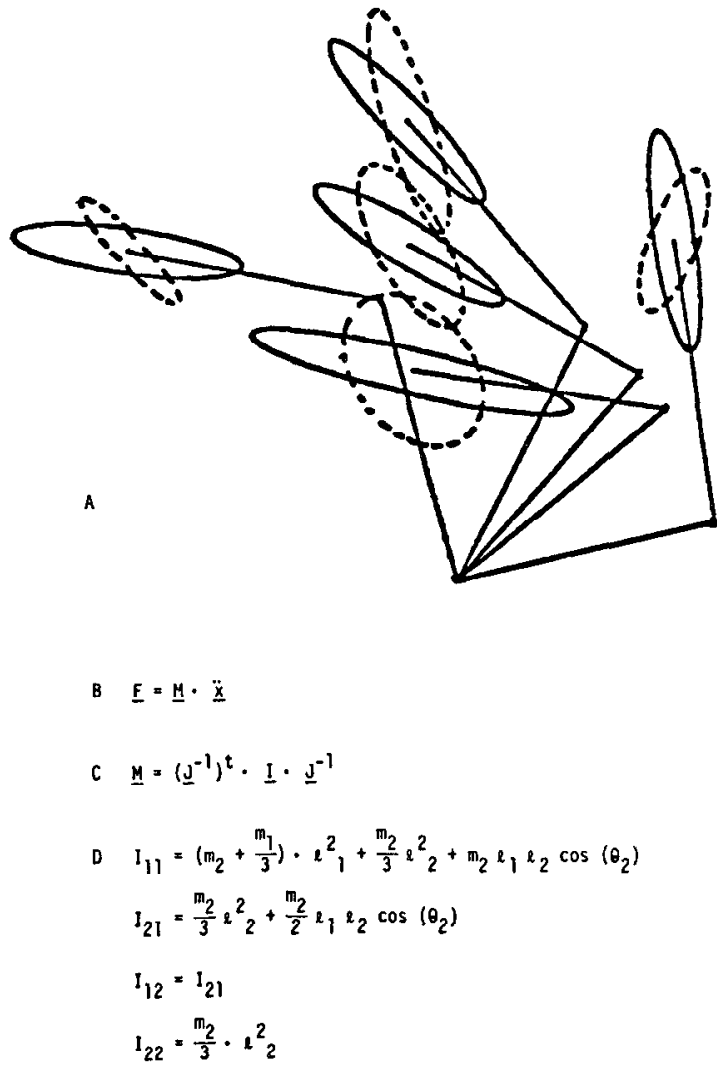

Figure 10. Comparison of stiffness and inertia. A, Stiffness (dashed lines) and inerita (solid lines) ellipses of subject PM in five work space locations. For each position the inertia ellipse shows the apparent mass of the hand along different directions, the maximum inertia corresponding to the major axis. $R$, Relation between force $(F)$ and acceleration ( $\ddot{x}$ ) vectors. $\underline{M}$ is the cartesian inertia represented by the ellipses. $C, \underline{M}$ can be computed from the joint inertia, 1 , by means of the inverse jacobian of the arm, $\underline{J}^{-1}, D$, Elements of the matrix: $\perp$ is the elbow angle, $m_{1}$ and $m_{2}$ are the upper arm and forearm masses (here $m_{1}=2.52 \mathrm{~kg}$ and $m_{2}=1.30 \mathrm{~kg}$ ), $\ell_{1}$ and $\ell_{2}$ are the upper arm and forearm lengths (here $\ell_{1}=33 \mathrm{~cm}$ and $\ell_{2}=32 \mathrm{~cm}$ ). 


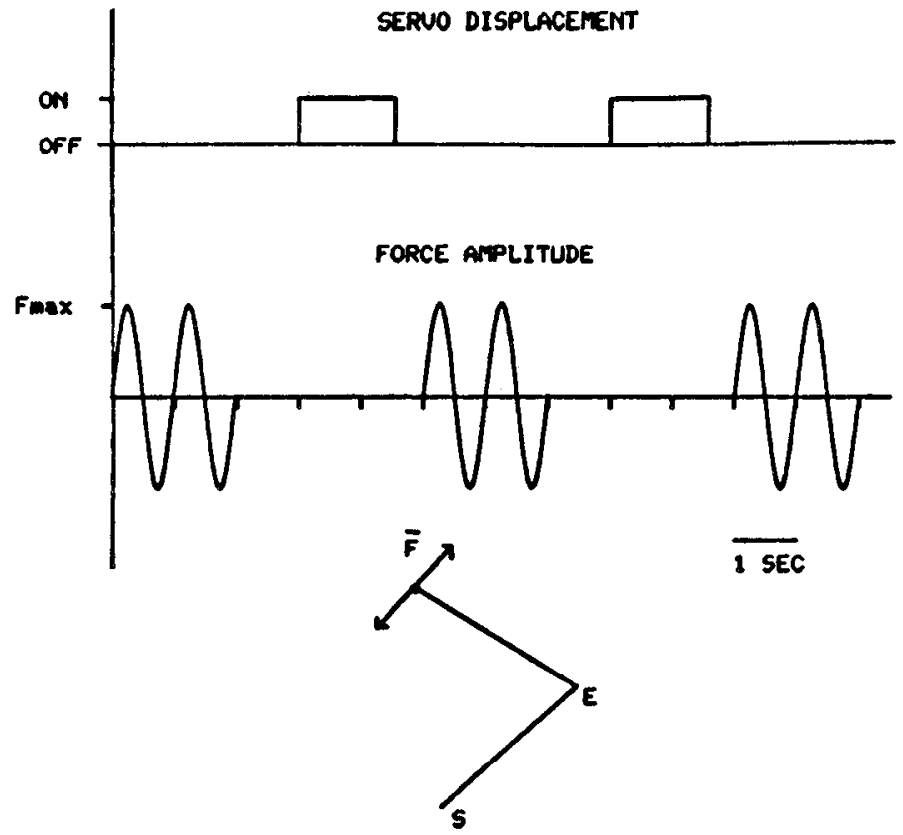

Figure $1 \%$. Paradigm for the force compensation experiment. A force of constant direction is applied to the hand of the subject. The arm is represented here by two segments ( $S$, shoulder, and $E$, elbow). The amplitude varies sinusoidally for a period of $2 \mathrm{sec}$. Then the force is removed and a servo displacement is applied for the measurement of the stiffness. A variation of this paradigm consisted in generating a rotating force of constant amplitude instead of constant direction.
MORMAL POSTURE

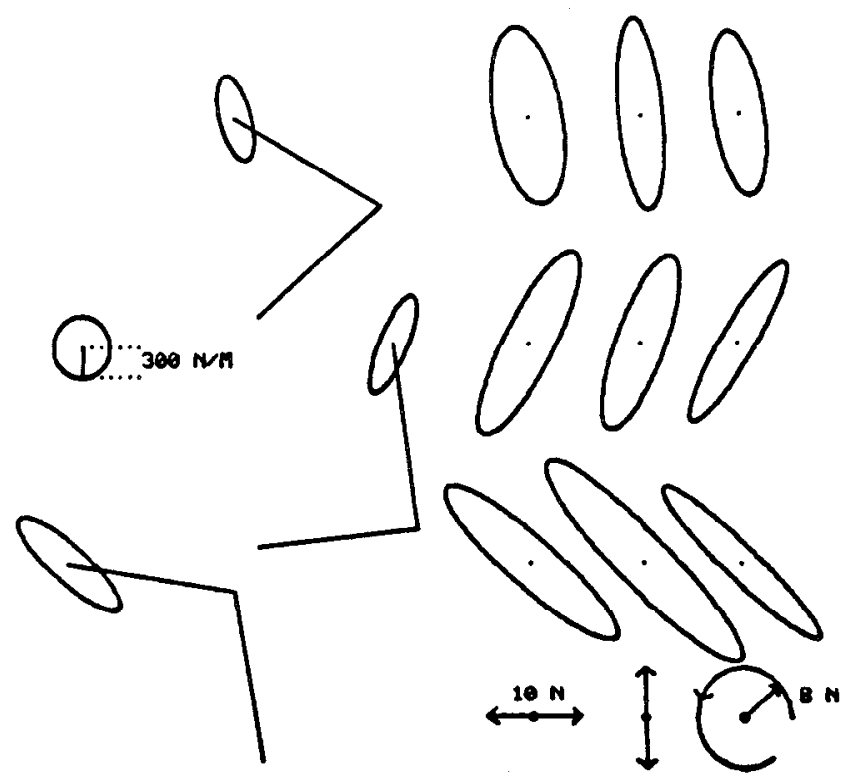

Figure 12. Changes in stiffness induced by force compensation. The stiffness ellipses measured in subject A during normal posture (left) are plotted here together with the stiffness ellipses resulting from force compensation. The ellipses on the right represent, for each arm configuration, the stiffness measured during the compensation of a force acting along the $x$ axis (left), a force acting along the $y$ axis (middle), and a rotating force of constant amplitude (right). The radial forces had a maximum amplitude of $10 \mathrm{~N}$ whereas the rotating ones had a constant amplitude of $8 \mathrm{~N}$.

this effect, we measured the stiffness of the hand while the subject kept it at the same target locations as before, but with a different shoulder abduction angle so that the arm no longer lay in the horizontal plane. The ellipses shown in dotted lines in Figure 13 indicate the results obtained in four target positions with the elbow close to the body. Comparing these results with those in solid lines, representing the stiffness in the usual planar posture, one can see that, in some of the positions, the ellipses have radically changed in shape and orientation. This finding indicates that the stiffness of the hand depends on the global configuration of the arm and not only on hand position.

\section{Discussion}

In the experiments described in this paper, we irivestigated the behavior of the arm and the hand in a multi-joint postural task. One of our primary goals was to characterize the spring-like behavior of the neuromuscular system. The idea that muscles display spring-like properties is not new; several recent studies have emphasized this point and have stressed that we can understand the organization of voluntary movements by studying the way in which the mechanical properties of the neuromuscular system set constraints upon the motor controller (Feldman, 1966; Hogan, 1980, 1984a, b; Bizzi et al., 1984). With this paper, we contribute to the growing understanding in this area by providing (1) a unique demonstration of the degree to which the neuromuscular system is spring-like; (2) a compact and powerful way of representing the size, shape, and orientation of spring-like behavior in a multi-joint system; (3) a description of the patterns and regularities in the stiffness observed during normal posture; (4) evidence of the ability of human subjects to modulate postural stiffness through coordinated synergistic muscle activation; (5) findings on the relation between inertial and elastic properties of the arm; and (6) a demonstration of the effect on postural stiffness of changing the arm configuration while the position of the hand is unchanged. Taken together, these findings represent a new view of the neural, mechanical, and geometric features subserving hand posture and movement.

Demonstration of spring-like behavior. The defining property of a spring is its ability to store elastic energy when stretched. In a onedimensional system, such as an individual muscle or a single joint, spring-like behavior requires the existence of only a single-valued, integrable relationship between force and displacement. In a multidimensional system, elastic behavior is present when the curl of the vector field of restoring forces due to displacement of the limb is zero. The absence of curl in turn requires the symmetry of the

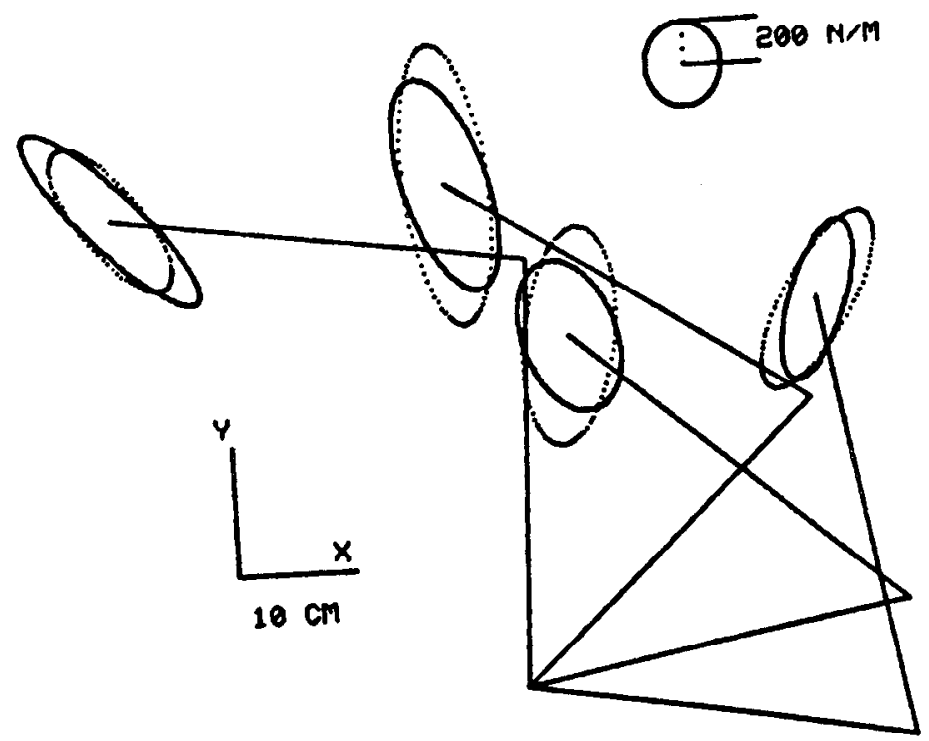

Figure 13. Changes in stiffness induced by changes in arm configuration. The stiffness ellipses shown in solid lines have been measured from subject A during planar arm posture in four positions. The stiffness ellipses in dotted lines have been measured in the same hand positions, but with a different shoulder abduction angle. The elbow was lowered and the upper arm was closer to the subject's body. 
stiffness tensor, i.e., the equality of the nondiagonal terms in the stiffness matrix. Our experimental results indicate that in two subjects the antisymmetry of the stiffness was not statistically significant. In another two subjects the antisymmetry was statistically significant, but the magnitude of the restoring forces due to the curl was a small fraction of the restoring forces due to the spring-like behavior. It follows that the neuromuscular system is predominantly spring-like.

Effects of intermuscular feedback. The small but statistically significant curl observed in some subjects is interesting in its own right. As delailed by Hogan (1985), this kind of behavior can be due only to an imbalance between the gains of the reflex pathways connecting different arm muscles. At the joint level this means that stretching the muscles around the elbow generates a torque around the shoulder which is greater (or less) than that generated around the elbow by stretching the shoulder muscles. However, the small value of the curl indicates that the action of intermuscular feedback, if any exists, is for the most part balanced and coordinated to preserve the spring-like behavior of the neuromuscular system.

Representation of stiffness. We have shown that, by displacing the hand in many different directions and measuring the steadystate restoring force that results, it is possible to determine the postural stiffness of the hand. In general, the stiffness associated with multi-joint arm posture is not a simple scalar quantity. In the multi-joint case the stiffness has a shape and a direction as well as a magnitude. In fact, the force displacement behavior of the multijoint limb defines a vector field of restoring forces. This field of forces can be expressed in a variety of coordinate systems-in end-point cartesian coordinates, or in joint coordinates, or as length-tension parameters of individual muscles - but in all cases the same physical phenomenon is being described and the fundamental relation bc tween displacement and force must remain unchanged.

The stiffness is a physical entity that may be represented in several ways. In this paper we have represented it in two ways: numerically, as a matrix of coefficients the values of which were obtained through a regression of force on displacement; and graphically, through the size, shape, and orientation of the ellipse associated with the symmetric component of the stiffness. Although the two are equivalent (see "Materials and Methods"), the graphical representation provides a "gestalt" and affords a qualitative understanding of the way in which the hand may interact with forces that could change its posture. Describing hand posture as an oriented stiffness ellipse helps us to determine which of the parameters of postural stiffness are subject to modulation and control by the CNS.

Patterns of stiffness variation. We found that the stiffness associated with hand posture varies substantially at different positions of the hand in the work space. Specifically, the shape and the orientation display a common pattern, which can be summarized as follows: the stiffness is more isotropic (circular) in proximal positions and more anisotropic (elongated) in distal positions; the direction of maximum stiffness is approximately oriented along a radial line joining the hand to the shoulder. Thus, in a displacement from right to left at constant hand-to-shoulder distance, the stiffness undergoes a counterclockwise rotation.

Several factors may contribute to such regular variations. First, the mechanical advantage of a force applied at the hand is a function of the elbow and shoulder joint angles and, as a consequence, the stiffness observed at the hand would be expected to change for different arm configurations. Second, the muscle tensions are subject to variations in their moment arms as the joint angles change in the work space, and the apparent stiffness of a given muscle may change with its length. As a consequence, the contribution of an individual muscle to the total stiffness of the arm is significantly different for different arm positions. Third, since the neural input to the muscles changes their spring-like properties (Rack and Westbury, 1969, 1974; Gottlieb and Agarwal, 1978), the observed variation in the stiffness may be due to the different levels of neural activation associated with different postures. In an effort to distinguish between these factors, we simulated the effect of a constant joint stiffness and found the simulated changes of endpoint stiffness to be qualitatively similar to those recorded from our subjects. This result clearly demonstrates the dominant influence of musculoskeletal geometry on the stiffness of the hand. However, the systematic difference between this simulation and our experimental data indicates that changes in other factors such as neural input and/or muscle properties contribite significantly to the determination of the hand stiffness. In particular, with a constant joint stiffness, the magnitude and shape of the elastic field increased very rapidly as the hand approached the boundaries of the work space. The actual hand stiffness observed in our subjects did not exhibit such a dramatic change as the arm approached positions away from the body.

When the stiffness was recorded in the same subject at intervals of days or monthis, there was a remarkable constancy in its shape and orientation. In contrast, it varied substantially in magnitude (up to $100 \%$ ). These variations can be attributed either to a change in the level of "arousal" of the subject or to the aftereffects of a prior experimental condition in which the subjects experienced postural disturbances. This variability in magnitude, coupled with the relative invariance in orientation and shape, is a strong indication that the increase in the motoneuronal activity which is responsible for the increased magnitude of the stiffness must be delivered in a well controlled way. A change in stiffness magnitude at constant shape and orientation can be achieved only by a uniformly scaled change in the individual stiffnesses of all the elastic elements. It suggests that the $\alpha$-motoneuron activities are subject to coordinative constraints resulting either from coupling among different motoneuronal pools or from supraspinal signals activating these pools. Our finding, then, indicates that contributions from ncural input may act in such a way as to counteract changes in hand stiffness produced by musculoskeletal geometry. A similar approach to the interaction between mechanical and neural constraints has recently been proposed for the interpretation of leg muscle activity during standing posture in humans (Nashner and McCollum, 1985).

Modulation of postural stiffness. When our subjects were asked to maintain stability of the hand against a force of constant (i.e., predictable) direction, we observed substantial changes in magnitude, but only minor changes in shape and orientation, a fact indicating that, for this task, the CNS does not increase the stiffness selectively in the direction in which more stability is required. However, further experiments are needed to assess whether this relative invariance of orientation and shape depends upon the action of geometrical and/or neural constraints. By geometrical constraints we refer to the muscle's length-tension properties and their moment arm. By neural constraints, we refer to the efferent and reflex synergist muscle activation.

Relationship between stiffness and inertia. The postural dynamics of the limb are due to the interplay of the impedance of the neuromuscular system (e.g., its stiffness, its viscosity) and the inertia of the timb. These quantities dictate the response of the limb to external disturbances. External "disturbances" will be encountered any time the limb performs a task, such as the use of a tool, which requires dynamic interaction between the limb and its environment, and modulating the impedance of the limb is one effective strategy for controlling dynamic interaction between the limb and a tool $(N$. Hogan, 1985). The inertia of a limb is fundamentally configuration dependent; the inertia of the hand, for example, changes substantially with its position in the work space. One possible form of postural coordination would be to modulate the stiffness of the neuromuscular system so that it bore a fixed relation to the inertia of the limb. This would result in an invariance in the postural dynamics of the limb despite the inevitable changes in the inertial behavior. The results of our preliminary investigation of the relation between stiffness and inertia are consistent with this idea.

Effect of arm configuration. Although our observations show that the predominant effect of changing neural input is to change the magnitude of the stiffness, changing the configuration of the arm 
while the hand remains in a given position has a profound effect on both the shape and the orientation of the stiffness. This suggests that an effective strategy for changing all parameters of the postural stiffness may be to combine changes in neural input to the muscles with changes in the configuration of the "extra," or redundant, degrees of freedom of the limb. Changes in arm configuration will also have a profound effect on other components of the neuromuscular impedance (such as viscosity) and, indeed, changing arm configuration is probably the only way the CNS can change the endpoint inertia of the limb (see Hogan, 1984b). From this point of view, it can be seen that the configuration of the limb should be regarded as one of the "command inputs" available to the CNS for controlling posture. The redundancy of the musculoskeletal system is usually regarded as a problem to be overcome by the CNS in coordinating limb movements (Bernstein, 1967); instead, the results reported in this paper show that it may offer the CNS alternative ways to control postural dynamic behavior.

\section{References}

Bernstein, M. (1967) The Co-ordination and Regulation of Movements, Pergamon Press, Oxford.

Bizzi, E., A. Polit, and P. Morasso (1976) Mechanisms underlying achievement of final head position. J. Neurophysiol. 39: 435-444.

Bizzi, E., P. Dev, P. Morasso, and A. Polit (1978) The effect of load disturbances during centrally initiated movements. J. Neurophysiol. 41: 542-556.

Bizzi, E., N. Accornero, W. Chapple, and N. Hogan (1982a) Arm trajectory formation. Exp. Brain Res. 46: 139-143.

Bizzi, E., W. Chapple, and N. Hogan (1982b) Mechanical properties of muscles: Implications for motor control. Trends Neurosci. 5: 395-398.

Bizzi, E., N. Accornero, W. Chapple, and N. Hogan (1984) Posture control and trajectory formation during arm movement. J. Neurosci. 4: 27382744.

Cooke, J. D. (1979) Dependence of human arm movements on limb mechanical properties. Brain Res. 165: 366-369.

Feldman, A. G. (1966) Functional tuning of the nervous system during control of movement or maintenance of a steady posture. III. Mechanographic analysis of the execution by man of the simplest motor tasks. Biophysics 11: 766-775.

Gottlieb, G. L., and G. C. Agarwal (1978) Dependence of human ankle compliance on joint angle. J. Biomech. 11: 177-181.

Hoffer, J. A., and S. Andreassen (1981) Regulation of soleus muscle stiffness in premammilary cats: Intrinsic and reflex components. J. Neurophysiol. 45: 267-285.

Hogan, N. (1980) Mechanical impedance control in assistive devices and manipulators. In Proceedings of the Joint Automatic Control Conference, San Francisco, California, Editors, pp. 000-000, Publisher, City.

Hogan, N. (1984a) Impedance control of industrial robots. Robots Comput. Integrated Mfg. 1: 97-113.

Hogan, N. (1984b) An organizing principlc for a class of voluntary movements. J. Neurosci. 4: 2745-2754

Hogan, N. (1985) The mechanics of multi-joint posture and movements. Biol. Cybern. 53: 1-17.

Houk, J. C., and W. Z. Rymer (1981) Neural control of muscle length and tension. In Handbook of Physiology. Section 1: The Nervous System, Vol. II, Part 2: Motor Control, V. B. Brooks, ed., pp. 257-323, American Physiological Society, Bethesda, MD.

Kelso, J. A. S. (1977) Motor control mechanisms underlying human movement reproduction. J. Exp. Psychol. 3: 529-543.

Kelso, J. A. S., and K. G. Holt (1980) Exploring a vibratory system analysis of human movement production. J. Neurophysiol. 43: 1183-1196.

Kendall, M. G., and A. Stuart (1961) The Advanced Theory of Statistics, Ed. 1. Hafner Publishing Co., New York.

Nashner, L. M., and G. McCollum (1985) The organization of human postural movement: A formal basis and experimental synthesis. Behav. Brain Sci., 8: $135-172$.

Nichols, T. R., and J. C. Houk (1976) Improvement in lineurity and regulation of stiffness that results from actions of stretch reflex. J. Neurophysiol. 39: $119-142$.

Polit, A., and $\mathrm{E}$. Bizzi (19/9) Characteristics of the motor programs underlying arm movements in monkeys. J. Neurophysiol. 42: 183-194.

Rack, P. M. H., and D. R. Westbury (1969) The effects of length and stimulus rate on tension in the isometric cat soleus muscle. J. Physiol. (Lond.) 204: 443-460

Rack, P. M. H., and D. R. Westbury (1974) The short range stiffness of active mammalian muscle and its effect on mechanical properties. J. Physiol (Lond.) 240; 331-350. 